\title{
Cultivating Desmodesmus armatus (Chod.) Hegew. in recirculating aquaculture systems (RAS) waste water
}

\author{
Larisa Cheban, Irina Malischuk, Mykhailo Marchenko
}

Received - 05 February 2015/Accepted - 02 August 2015. Published online: 31 October 2015; Inland Fisheries Institute in Olsztyn, Poland Citation: Cheban L., Malischuk I., Marchenko M. 2015 - Cultivating Desmodesmus armatus (Chod.) Hegew. in recirculating aquaculture systems (RAS) waste water - Arch. Pol. Fish. 23: 155-162.

\begin{abstract}
The possibility of using RAS waste water as a cultivation medium for Desmodesmus armatus (Chod.) Hegew. was investigated. Algal biomass increased continuously reaching its maximum on the day 40 of cultivation. D. armatus culture was characterized by a maximum content of total proteins of $23 \%$ on day 40 of cultivation. At the same time, 17 proteinogenic amino acids, including 9 replaceable and 8 essential acids, were detected and identified in D. armatus biomass. Among these amino acids, maximum contents of asparagine and glutamine were noted. A gradual increase in the number of total photosynthetic pigments until day 40 of cultivation was also observed. On day 40 , the content of chlorophyll $a, b$, and carotenoids in D. armatus cells was maximal at $11.17,7.07$, and 12.05 $\mathrm{mg} \mathrm{g}^{-1}$ dry weight, respectively. Therefore, cultivating microalgae $D$. armatus in RAS waste water permits obtaining an active growing culture that is characterized by a constant increase of biomass and high nutrient contents.
\end{abstract}

Keywords: biomass, chlorophyll $a, b$, carotenoids, recirculating water system.

\footnotetext{
L. Cheban [ $\left.\Xi^{\circ}\right]$, I. Malischuk, M. Marchenko

Department of Biochemistry and Biotechnology, Yuriy Fedkovych Chernivtsi National University, Ukraine e-mail: larisa.cheban@mail.ru
}

\section{Introduction}

The advantage of biotechnology for the controlled cultivation of photosynthetic organism is the low loss of valuable biomass, which is used both in natural and processed conditions (Zolotareva et al. 2008). The high protein content, including essential amino acids, and carbohydrates, lipids, and pigments in numerous microalga species and their lability under various cultivation conditions render it possible to use them as sources of biologically active substances and to manage their biosynthesis in the required directions (El-shimy and Ismail 2007). Knowledge about the mechanisms of microalgal adaptation to various cultivation conditions and the chemical components of culture media is not only of theoretical interest for producing highly-productive strains, but also of great practical importance, particularly for the development of modern technologies for the manufacture of productive feeds and premixes (Ladygina 2007). Simultaneously, is important that the algal mass obtained is non-toxic. Thus, possibilities for the comprehensive use of microalgae requires actively screening highly productive strains and optimizing culture conditions. This is because changes in the functional activity and nutrient composition of microalgae during dynamic cultivation are possible during the formation of population structures with

\footnotetext{
(c) Copyright by Stanisław Sakowicz Inland Fisheries Institute in Olsztyn.

(C) 2015 Author(s). This is an open access article licensed under the Creative Commons Attribution-NonCommercial-NoDerivs License (http://creativecommons.org/licenses/by-nc-nd/3.0/).
} 
the requisite biosynthetic properties (Zolotareva et al. 2008).

For most algal representatives, the general guidelines were developed for cultivation under laboratory conditions depending on the purpose of the experiment; these included the choice of medium, the inoculation of producer's culture into the culture medium, and accumulative cultivation over a certain period. Thus, the nutritive elements and different sources of carbon nutrition (various forms of nitrogen $\left[\mathrm{NH}^{4+}, \mathrm{NO}^{3-}, \mathrm{NO}^{2-}\right]$, phosphorus and others) were necessary for increasing the algal biomass (Nunez et al. 2001, Mallick 2002, Denisov and Zhuikov 2007). Common approaches in biotechnology include using mixtures of macro- and microelements that contain all the necessary components as culture media (Zolotareva et al. 2008). However, artificial media are quite expensive. On the other hand, the yield of algae biomass with improved productive characteristics can be achieved only by changing the composition of the culture medium (Chubchikova et al. 2013). So, it is necessary to find alternative culture media that, on the one hand, meet the mineral component needs of algal cultures and that, on the other, help to reduce the technological costs of producing algal biomass (Golub 2013, Mamedova et al. 2013). Therefore, the physiological characteristics of algae, such as active photosynthesis, the ability to directly utilize nitrogen and phosphorus ions and other biogenic elements, and to withstand the high concentrations of toxic substances and metabolites permits using waste water of various origin as culture media (Makarova et al. 2009, Woertz et al. 2009). This approach allows, on the one hand, to reduce the expense of cultivation systems and, on the other, to clean waste water of nutrients (Gorbunova and Zubko 2009). The waste water from recirculating aquaculture systems (RAS) is rich in nutrients, particularly in various forms of nitrogen, and can be considered as a culture medium.

The possibility of cultivating the blue-green algae Anabaena hassalii in RAS waste water has been demonstrated previously (Cheban et al. 2014). However, protococcus algae are one of the most active agents used to clean waste waters (Samorí et al.
2013). The phototrophic ability of the $D$. armatus algal culture can enrich the aquatic environment with oxygen that accelerates oxidation processes and the mineralization of organic compounds. In addition, $D$. armatus biomass can be used as a source of proteins, amino acids, vitamins, polyunsaturated fatty acids, and pigments, including carotenoids (Sharapova et al. 2012). This permits using the $D$. armatus biomass as a source of complex nutrients and for the needs of aquaculture (Cetin and Mert 2006). Consequently, D. armatus culture productivity was estimated using RAS waste water as the culture medium.

\section{Materials and Methods}

The research was conducted on an algological pure culture of green alga, Desmodesmus armatus (Chod.) Hegew. (IBASH-A), obtained from the collection at the M.G. Kholodny Institute of Botany of the National Academy of Sciences of Ukraine. RAS waste water was used as the culture medium. The water was taken from a mechanical filter, poured onto aliquots, autoclaved at $121^{\circ} \mathrm{C}$ for $30 \mathrm{~min}$, and standardized at a pH index of 7.5-8 with total mineralization of 495 \pm 5 ppm (Cheban et al. 2014). Fitzgerald's medium No 11 with Zehnder's and Gorham's modifications was used as the comparative medium (Zolotareva et al. 2008). The cultivation was carried out in $500 \mathrm{~mL}$ Erlenmeyer's flasks at a temperature of $21 \pm 2^{\circ} \mathrm{C}$, illuminated with 2500 lux fluorescent lamps for a 16-hour photoperiod (Gaysina et al. 2008). The inoculation was conducted at a ratio of inoculum:culture medium of 1:10. The physicochemical parameters of the culture medium were monitored throughout cultivation ( $\mathrm{pH}$, Ionometry U-160 MU; total mineralization, Water Quality Tester COM-100 conductivity meter). Biomass was determined by culture density using the optical index at $750 \mathrm{~nm}$ on SF-46. The transition from units of optical density (D750) to the value of absolutely dry biomass (ADB) was done with empirical coefficient $\mathrm{k}$ :

$$
A D B=k \times D_{750} .
$$


Coefficient $\mathrm{k}(\mathrm{k}=\mathrm{g} \times$ absorbance units $/ \mathrm{l})$ for the D. armatus culture was determined experimentally in three independent replicates (Gorbunova and Zhondareva 2012).

The microalgae suspension was centrifuged at 8000 g per 15 minutes with Biofuge Stratos (Heraeus Instruments). The biomass was disintegrated on UZDN-2T after centrifugation. The content of proteins (Vohnivenko et al. 2009), the qualitative and quantitative amino acid composition, the total content of chlorophyll $a, b$ (Campbell et al. 1998), and total carotenoids (Sánchez et al. 2008) were determined in crude cells. All calculations were performed on dry weights. Amino acid hydrolysis was performed with concentrated hydrochloric acid at a temperature of $106^{\circ} \mathrm{C}$ for 24 hours. The complete composition of amino acids was analyzed on an automatic T-339 amino acid analyzer (Mikrotechna, Czech Republic) at the O. Palladin Institute of Biochemistry of the National Academy of Sciences of Ukraine. The spectra of pigments in the acetone extracts were measured by spectrophotometry on SF-46 in the wavelength range of $400-800 \mathrm{~nm}$. The calculations of pigment concentrations were conducted according to formulas (Jeffrey and Humphrey 1975) by optical density indexes at wavelengths

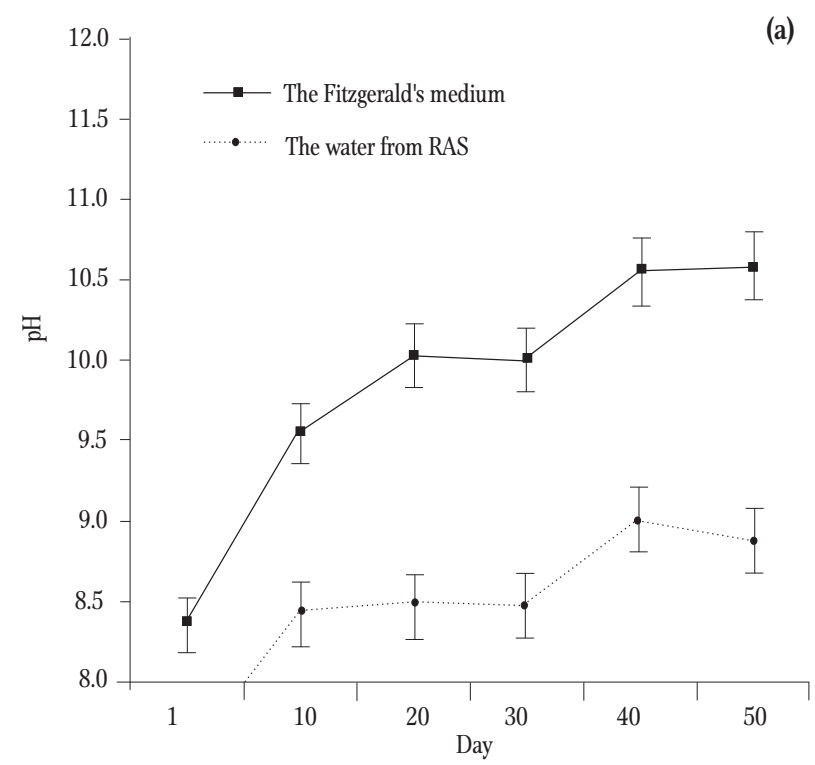

corresponding to maxima absorption of chlorophyll $a, b$, and total carotenoids.

\section{Statistical Analysis}

Mean values were considered significantly different at $\mathrm{P} \leq 0.01$ according to Student's criterion. The results were analyzed statistically with Microsoft Excel software according to generally accepted methods.

\section{Results}

The growth of biomass indices was observed against a background of exponential increases in total mineralization up to day 10 of cultivation in both the RAS waste water and the artificial medium. However, beginning from day 20 of cultivation, the general mineralization indexes returned to the initial levels. A slight increase in total mineralization was correlated with changes in $\mathrm{pH}$. An increase in $\mathrm{pH}$ up to day 40 was noted, but it did not exceed pH 9.0 during cultivation in waste water (Fig. 1). Low culture growth was noted during the first days of $D$. armatus cultivation in both culture media, which was obviously caused by the microalgae adapting to the new

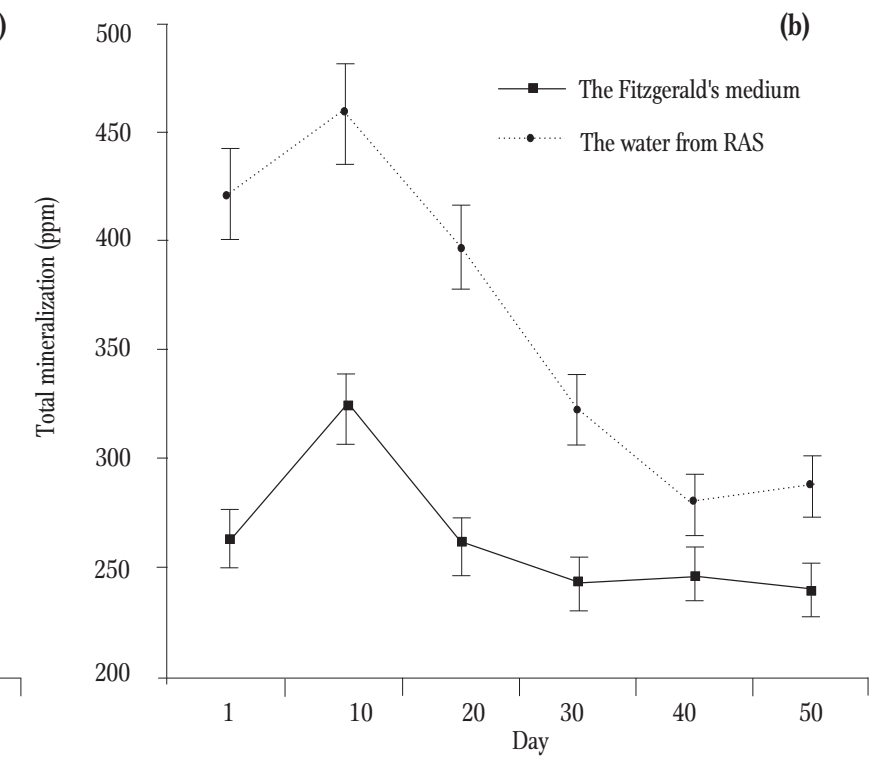

Figure 1. The changes of $\mathrm{pH}$ (a) and total mineralization (b) in control (Fitzgerald's medium) and cultures with water from RAS of D. armatus. 

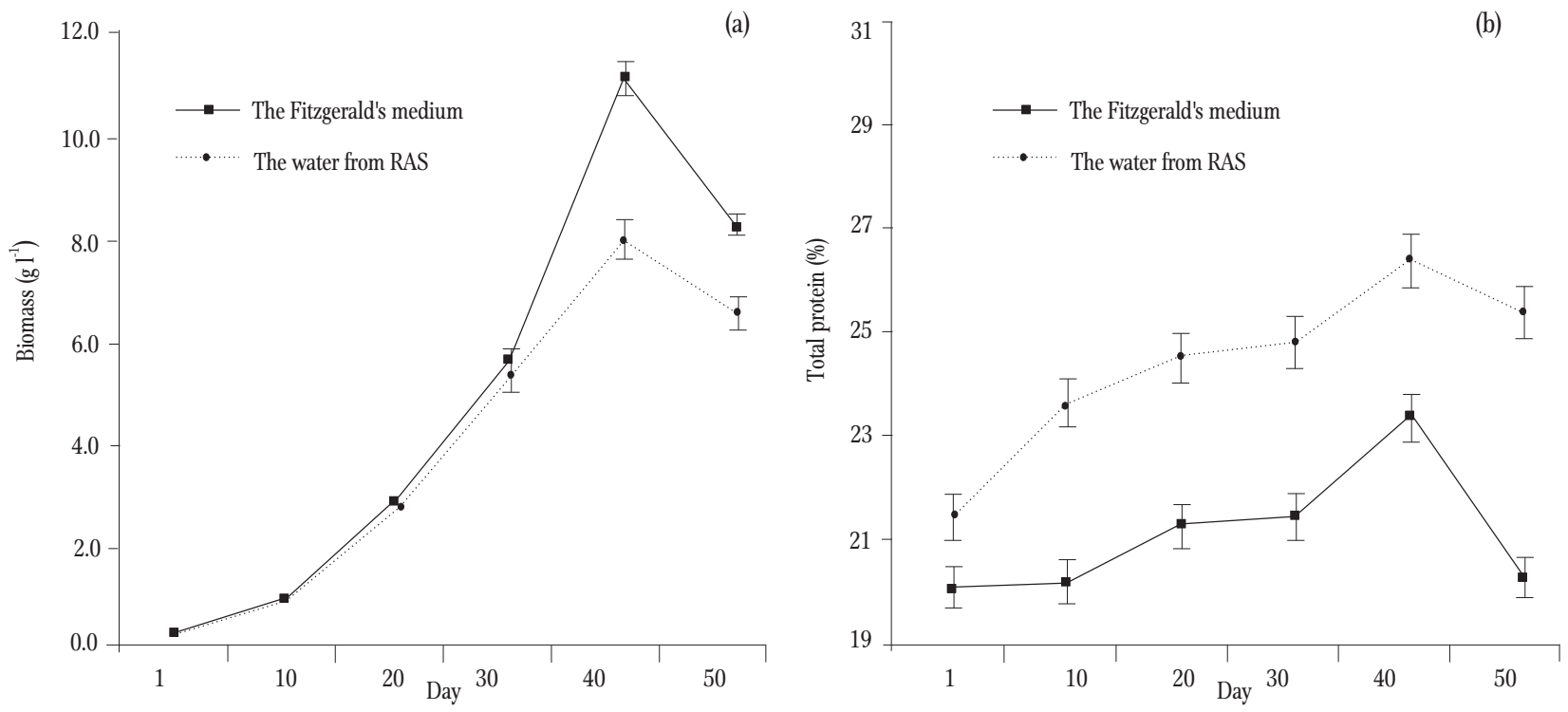

Figure 2. The changes of biomass (a) and total protein (b) in control (Fitzgerald's medium) and cultures with water from RAS of D. armatus.

Table 1

Amino acid composition of $D$. armatus biomass (mean $\pm \mathrm{SD}$ )

\begin{tabular}{lll}
\hline \hline Amino acid & RAS water $\left(\mathrm{mg} \mathrm{g}^{-1}\right)$ & Fitzgerald's medium $\left(\mathrm{mg} \mathrm{g}^{-1}\right)$ \\
\hline \hline Lysine (Lys) & $0.148 \pm 0.008$ & $0.106 \pm 0.014$ \\
Histidine (His) & $0.038 \pm 0.003$ & $0.021 \pm 0.009$ \\
Arginine (Arg) & $0.146 \pm 0.009$ & $0.086 \pm 0.011$ \\
Asparagine (Asp) & $0.322 \pm 0.030^{\mathrm{a}}$ & $0.243 \pm 0.021^{\mathrm{b}}$ \\
Threonine (Thr)) & $0.160 \pm 0.010$ & $0.119 \pm 0.004$ \\
Serine (Ser) & $0.153 \pm 0.018$ & $0.117 \pm 0.031$ \\
Glutamic acid (Glu) & $0.350 \pm 0.008^{\mathrm{a}}$ & $0.258 \pm 0.030^{\mathrm{b}}$ \\
Proline (Pro) & $0.139 \pm 0.045$ & $0.116 \pm 0.006$ \\
Glycine (Gly) & $0.183 \pm 0.007$ & $0.152 \pm 0.023$ \\
Alanine (Ala) & $0.245 \pm 0.016$ & $0.207 \pm 0.026$ \\
Cysteine (Cys) & $0.010 \pm 0.002$ & $0.006 \pm 0.001$ \\
Valine (Val) & $0.084 \pm 0.013$ & $0.067 \pm 0.017$ \\
Methionine (Met) & $0.008 \pm 0.001$ & $0.006 \pm 0.001$ \\
Isoleucine (Ile) & $0.045 \pm 0.005$ & $0.036 \pm 0.007$ \\
Leucine (Leu) & $0.189 \pm 0.024^{\mathrm{a}}$ & $0.152 \pm 0.043^{\mathrm{b}}$ \\
Tyrosine (Tyr) & $0.083 \pm 0.021$ & $0.058 \pm 0.018$ \\
Phenylalanine (Phe) & $0.114 \pm 0.038$ & $0.088 \pm 0.011$ \\
Total amino acids & $2.416 \pm 0.048$ & $1.840 \pm 0.037$ \\
\hline \hline
\end{tabular}

Data in rows with different letter indexes differ significantly statistically $(\mathrm{P} \leq 0.01)$

conditions (Fig. 2). From day 10, the amplification of growing processes in the investigated culture was observed. The most intensive growth of $D$. armatus biomass was noted between days 10 and 40 of cultivation, when its maximal productivity was also noted. Beginning from day 40 of the experiment, the amount of biomass in both media starts to decrease because of the algal culture transition to the degradation phase. Parallel with biomass growth, biosynthesis is activated in the exponential phase. 

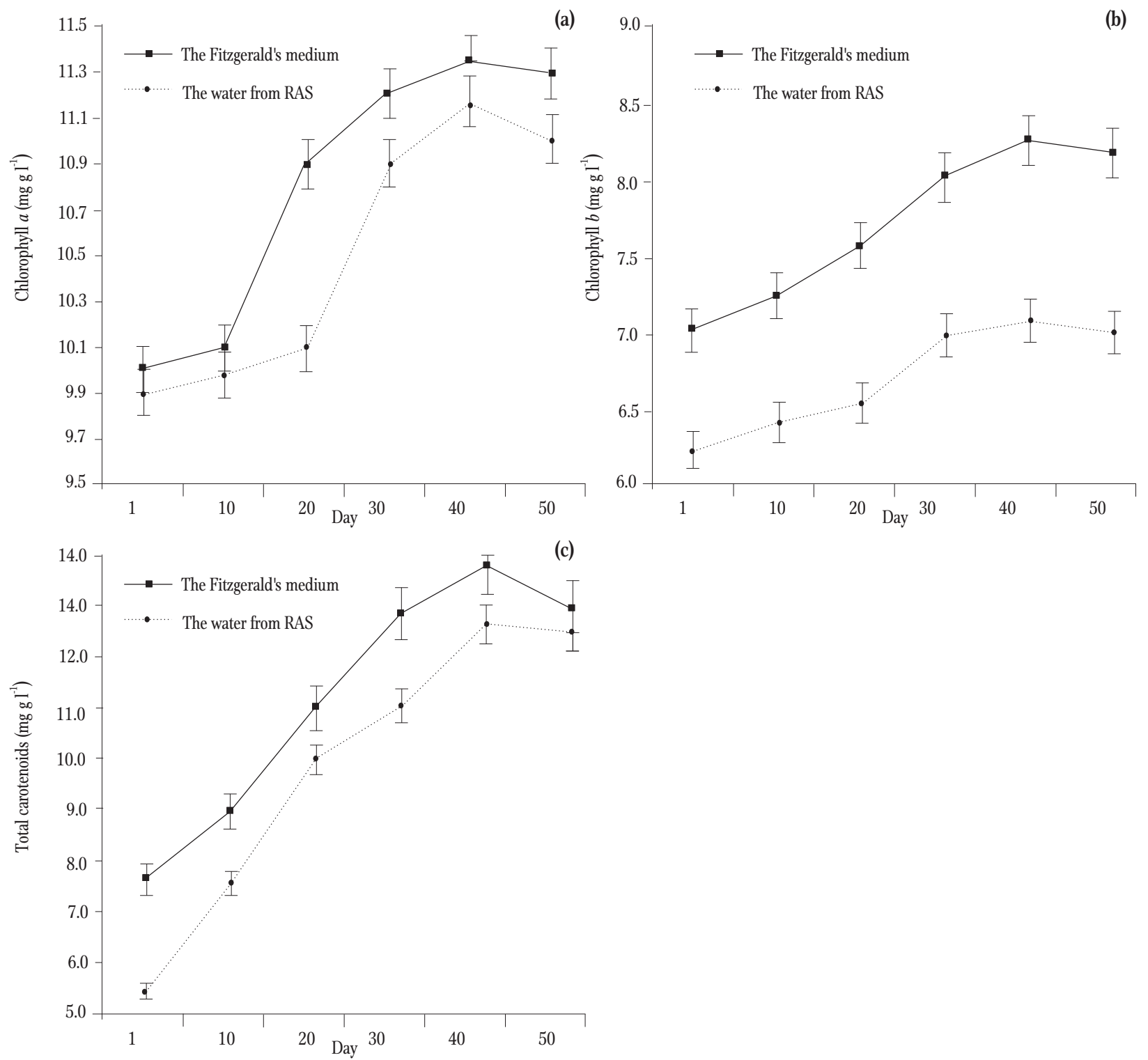

Figure 3. The changes of chlorophyll $a$ (a), chlorophyll $b(\mathrm{~b})$, and total carotenoids (c) in control (Fitzgerald's medium) and cultures with water from RAS of $D$. armatus.

A gradual increase of protein content in D. armatus cells was noted from days 10 to 40 of cultivation in both RAS water and in Fitzgerald's medium, and on day 40 of the experiment it reached maximal values of 26 and 23\%, respectively. Simultaneously, 17 proteinogenic amino acids, including 9 replaceable and 8 essential ones, were detected and identified in D. armatus biomass cultivated in waste water (Table 1). Among the essential amino acids, tryptophan was absent. The spectrum of amino acids in D. armatus biomass that was cultivated in both culture media did not differ in qualitative composition, but did so with regard to quantitative indicators.

The highest possible content of aspartic and glutamic acids was observed in $D$. armatus biomass that grew in both media with amounts ranging from 0.320 to $0.350 \mathrm{mg} \mathrm{g}^{-1}$ in waste water and from 0.240 to $0.260 \mathrm{mg} \mathrm{g}^{-1}$ in Fitzgerald's medium. Among the essential amino acids, the highest content was of leucine, but the amount of it in the D. armatus biomass cultivated in waste water was $0.189 \mathrm{mg} \mathrm{g}^{-1}$ in comparison to $0.152 \mathrm{mg} \mathrm{g}^{-1}$ in Fitzgerald's medium. 
The productivity of algae cultures was also estimated using indicators of pigment accumulation, in particular chlorophyll $a, b$, and carotenoids. The dependence of photosynthetic efficiency on mineral nutrition elements was determined by their necessity in the formation of both the photosynthetic apparatus and its renewal during the lifetime of the culture. During D. armatus cultivation in waste water, a gradual increase in the amounts of chlorophyll $a$ and $b$ was noted, and they reached maximal values at the stationary phase of culture growth (Fig. 3). Beginning on day 10 of cultivation, a gradual increase in the amount of pigments was observed. On day 40 of the experiment, the chlorophyll $a$ and $b$ content in $D$. armatus cells was at the highest possible levels of 11.17 and $7.07 \mathrm{mg} \mathrm{g}^{-1}$ of dry weight of chlorophyll $a$ and $b$, respectively. Upon further cultivation in both culture media, a slight decrease in the amount of these pigments was noted.

The maximal content of carotenoids matches the stationary phase of D. armatus culture growth. Thus, on day 40 of cultivation this parameter reached 12.05 and $13.79 \mathrm{mg} \mathrm{g}^{-1}$ of dry weight during cultivation of algal cultures in waste water and in synthetic medium, respectively. Upon further cultivation, the amount of carotenoids in D. armatus cells increased slightly, but it does not significantly differ from the maximal values that were noted on day 40 of cultivation. The pigment contents in D. armatus cells depended on both the growth phase and the cultivation conditions. During this time, the dynamics of carotenoid accumulation correlated with dynamics that are observed while determining the amount of chlorophyll $a$ and $b$ and the biomass amount. Therefore, the degree of biomass increase in RAS water was slightly lower compared to the biomass increase in Fitzgerald's medium. This regularity was also noted when studying the accumulation indexes of carotenoids and photosynthetic pigments, particularly chlorophyll $a$ and $b$. Simultaneously, the quantitative content of total proteins in D. armatus algal cells that were grown in waste water was 3\% higher in comparison to the culture grown in the synthetic medium. This also applies to the study of the quantitative characteristics of individual amino acids.

\section{Discussion}

Representatives of Desmodesmus sp. are used for obtaining various products from natural and processed algal masses that are rich in vitamins, proteins, carbohydrates, and macro- and microelements; they are also used in aquaculture as food for mollusks, fish, and crustaceans (Becker 2007, Ladygina 2007). Desmodesmus sp. species are considered to be one of the most promising sources of raw materials thanks to their ability to grow in different types of waste waters and their high productivity (Imran et al. 2014). Algal biomass growth rates are dependent primarily on culture medium composition and cultivation conditions (light intensity, photoperiod, temperature, etc.). Regulating the mineral elements provided to microalgae is also necessary in order to achieve highly productive growing cultures (Chubchikova et al. 2013). Therefore, analyzing changes in cultivation parameters, including $\mathrm{pH}$ and total mineralization is quite important (Imran et al. 2014). By continuously monitoring the physicochemical indicators of culture media, it is possible to establish the optimal duration of $D$. armatus cultivation that lasts for 40 days.

In the first days of $D$. armatus cultivation in both culture media, low growth activity was noted, which is obviously caused by the microalgae adapting to new conditions. The duration of the lag phase was five days. The activation of growth processes was observed from day 10 of cultivation, and in this period the biomass of the algae doubled. These observations do not contradict data obtained for other species of the genus, particularly Scenedesmus obliquus (Toyub et al. 2008). The intensification of growth processes in both culture media from days 10 to 30 is an indication of the culture adapting excellently to the new conditions in the waste water. This trend was facilitated by the availability of mineral nutrition components (Oturyna et al. 2010).

After day 30, the biomass in the waste water medium was lower in comparison with that in Fitzgerald's medium. This was because the waste water was exhausted faster since its chemical composition differed from that in the artificial medium. The protein 
content of the algal mass cultivated in waste water is higher compared to that in algal mass from Fitzgerald's medium. Meanwhile, the quantitative characteristics of the amino acid composition was similar for both media, and were typical of the content of total proteins. In this case, one of the key factors was the quantity and form of available nitrogen (González López et al. 2010, Crofcheck et al. 2012). Thus, there were no differences in the qualitative content of the amino acids in the algal masses between the two culture media.

Each type of algae contains a complex of pigments in differing ratios. Carotenoids (astaxanthin, canthaxanthin, keto-carotenoids) (Pirastru et al. 2012) and chlorophyll $a$ and $b$ (Sánchez et al. 2008) accumulate in the biomass of Desmodesmus sp. species. The pigment dynamics in algae culture growth cycles stems from the features of accumulative cultivation. So, during long-term cultivation, the increasing of cell numbers leads to the exhaustion of the culture medium and shortages of certain minerals. At the same time, microalgae cultures are shaded by their own high densities, which gradually leads to the features of monoculture $D$. armatus development that was established previously. Starting on day 40 of the experiment, the algae culture began approaching the degradation phase, and its growth and productivity were limited by decreases in available nutrients and the accumulation of metabolic products in the culture fluid.

Cultivating D. armatus in RAS waste water permits obtaining active growing cultures characterized by constant biomass growth and high contents of total proteins, complex proteinogenic amino acids, and the main photosynthetic pigments. At the same time, the effectiveness of cultivation in RAS waste water in not significantly different from that in artificial Fitzgerald's medium. The culture obtained in this way can be used for further processing or transferred into fresh culture media. Using RAS waste water as a culture medium, on the one hand, helps to reduce significantly the costs of algal mass biotechnology and, on the other, it cleans the water of nutrients.

\section{Conclusion}

Therefore, RAS waste water can be used as a culture medium for D. armatus cultivation. This approach facilitates obtaining active-growing cultures that are characterized by constant biomass growth and high contents of total proteins and the main photosynthetic pigments.

Author contributions. M.M., L.C. and I.M. conceived of and implemented the experiment; I.M. and L.C. performed the experiment, analyzed the date and wrote the paper.

\section{References}

Becker E. 2007 - Micro-algae as a source of protein Biotechnol. Adv. 25: 207-210.

Campbell D., Hurry V., Clarke A., Gustafsson P., Öquist G. 1998 - Chlorophyll fluorescence analysis of cyanobacterial photosynthesis and acclimation Microbiol. Mol. Biol. Rev. 62: 667-683.

Cetin A.K., Mert N. 2006 - Growth rate of Scenedesmus acutus (Meyen) in cultures exposed to trifluralin - Pol. J. Environ. Stud. 15: 631-633.

Cheban L., Malischuk I., Lisak V., Marchenko M. 2014 - Efficiency growing of Anabaena hassalii (Kutz.) Wittr. under different culture conditions - Biological systems 6 (2): 145-149.

Chubchikova I.N., Minyuk G.C., Drobetskaya I.B., Dantsyuk N.W. 2013 - Optimization of culture medium for growing of microalgae Scotiellopsis rubescens Vinatz. (Chlorophyseae) - The notes of Taurian National University name of V. I. Vernadskij, Series «Biology. Chemistry» 26(65) 4: 196-205.

Crofcheck C., Shea A., Montross M., Crocker M., Andrews R. 2012 - Influence of media composition on the growth rate of Chlorella vulgaris and Scenedesmus acutus utilized for $\mathrm{CO}_{2}$ mitigation - J. Biochem. Tech. 4: 589-594.

Denisov A., Zhuikov V. 2007 - Waste water treatment in open reservoirs from organic and mineral impurities by using of algae - Achievements of science and technics APC 12: 54-57.

El-shimy A.A., Ismail A.G. 2007 - Accumulation of amino acids in Anabaena oryzae in response to sodium chloride salinity - J. Appl. Sci. Res. 3: 263-266.

Gaysina L., Fazlutdinova A., Kabiro R. 2008 - Modern methods of isolation and cultivation of microalgae - Ufa, BGPU, $152 \mathrm{p}$. 
Jeffrey S., Humphrey G. 1975 - New spectrophotometric equations for determining chlorophylls $a, b, c 1$ and $c 2$ in higher plants, algae and natural populations - Biochem. Physiol. Pflanzen 167: 191-194.

Golub N. 2013 - Microalgae cultivation using wastes - European advanced technology magazine 6/10(66): 4-9.

González López C.V, Cerón García M., Fernández F.G.A, Bustos C.S, Chisti Y., Sevilla J. M.F. 2010 - Protein measurements of microalgal and cyanobacterial biomass Bioresource Technology 101(19): 7587-7591.

Gorbunova S., Zhondareva I. 2012 - About efficiency using of microalgae in industrial biotechnology for the purpose of reclamation of water environment and for receiving of feed for different sectors of agriculture - Condition and prospects of aquaculture 23: 114-119 (in Russian).

Gorbunova S., Zubko V. 2009 - Using of Scenedesmus bijugatus (Lageth) for biological refining of waste water Proc. V Conf., 8-9.10. 2009, Sevastopol: 105-110 (in Russian).

Imran P., Kaumeel C., Basil G., Tonmoy G., Chetan P., Rahulkumar M., Sandhya M. 2014 - Nitrogen stress triggered biochemical and morphological changes in the microalgae Scenedesmus sp. CCNM 1077 - Bioresource Technology 156: 146-154.

Jeffrey S.W, Humphrey G. 1975 - New spectrophotometric equations for determining chlorophylls $a, b, c_{1}$ and $c_{2}$ in higher plants, algae and natural phytoplankton Biochem. Physiol. Pflanzen 167: 191-194.

Ladygina L. 2007 - Microalgae as food items for larvae of mussels and oysters - Autoreferat $\mathrm{PhD}$ thesis, Sevastopol, p. 24. (in Russian).

Makarova E., Oturina I., Sidyakin A. 2009 - Applied aspects using of microalgae - the inhabitants of aquatic ecosystems - Optimization and protection of Ecosystems, Simferopol, TNU, 20: 120-133 (in Russian).

Mallick N. 2002 - Biotechnological potential of immobilized algae for wastewater N, P and metal removal: a review BioMetals 15: 377-390.

Mamedov F., Nikolska A., Efremenko E. 2013 - Research of possibility of using waste water for microalgae biomass accumulation - Herld Kuzbss State Technical University 1: 113-115.
Nuńez V.J., Voltolina D., Nieves M., Pińa P., Medina M., Guerrero M. 2001 - Nitrogen budget in Scenedesmus obliquus cultures with artificial wastewater - Bioresour Technol. 78(2): 161-164.

Oturyna I., Makarova E., Sidyakin A. 2010 - Features dynamics of basic pigments of photosynthesis and accumulation of biomass in microalgae Scenedesmus sp. - representative of microalgoflora-freshwater ecosystems - Optimization and protection of Ecosystems, Simferopol, TNU, 21: 84-91 (in Russian).

Pirastru L., Darwish M., Lam Chu F., Perreault F., Sirois L., Sleno L., Popovic R. 2012 - Carotenoid production and change of photosynthetic functions in Scenedesmus sp. exposed to nitrogen limitation and acetate treatment $-\mathrm{J}$. Appl. Phycol. 24: 117-124.

Samorí G., Samorí C., Guerrini F., Pistocchia R. 2013 Growth and nitrogen removal capacity of Desmodesmus communis and of a natural microalgae consortium in a batch culture system in view of urban waste water treatment: Part I - Water Res. 47: 791-801.

Sánchez-Macías M.D., Serrano C.M., Rodríguez M.R., de la Ossa E.M., Lubían L.M., Montero O. 2008 - Extraction of carotenoids and chlorophyll from microalgae with supercritical carbon dioxide and ethanol as cosolvent - J. Sep. Sci. 31: 1352-1362.

Sharapova I., Shubkov A., Mykhailova E., Volodyn V. 2012 Investigation and use of microalgae for bioremediation of water environments - Innovations in science 13: 143-146 (in Russian).

Toyub M.A., Miah M.I., Habib M.A.B., Rahman M.M. 2008 Growth performance and nutritional value of Scenedesmus obliquus cultured in different concentrations of sweetmeat factory waste media - J. Anim. Sci. 37: 86-93.

Vohnivenko L., Yevtushenko M., Shevryakov M. 2009 - Biochemistry aquatic - Kherson, Oldi-plyus, 536 p. (in Russian).

Woertz I., Feffer A., Lundquist T., Nelson Y. 2009 - Algae grown on dairy and municipal wastewater for simultaneous nutrient removal and lipid production for biofuel feedstock - J. Environ. Eng. 135: 1115-1122.

Zolotareva O., Shnyukova E., Sivash O., Mihaylenko N. 2008 - Prospects of use of microalgae in biotechnology - Kyiv, Alterpres, 234 p. (in Ukrainian). 